\title{
Antitumor effect of antiplatelet agents in gastric cancer cells: an in vivo and in vitro study
}

\author{
Jota Mikami $^{1}$ - Yukinori Kurokawa ${ }^{1}$. Tsuyoshi Takahashi ${ }^{1} \cdot$ Yasuhiro Miyazaki $^{1}$ • \\ Makoto Yamasaki $^{1} \cdot$ Hiroshi Miyata ${ }^{1}$ Kiyokazu Nakajima ${ }^{1} \cdot$ Shuji Takiguchi $^{1}$. \\ Masaki Mori $^{1}$ - Yuichiro Doki ${ }^{1}$
}

Received: 3 July 2015/Accepted: 1 October 2015/Published online: 20 October 2015

(C) The International Gastric Cancer Association and The Japanese Gastric Cancer Association 2015

\begin{abstract}
Background The antitumor effects of antiplatelet agents in gastric cancer cells are not well known. In this study, the possibility of gastric cancer treatment with an antiplatelet agent, mainly aspirin, was examined both in vivo and in vitro.

Methods For in vivo experiments, tumor-bearing mice were treated by an antiplatelet antibody or aspirin, and the tumor growth was compared. For in vitro experiments, human gastric cancer cell lines were used to confirm the cancer cell growth and inhibition by reducing the platelet count or using aspirin. We also examined several cytokines by using an ELISA assay and conducted microRNA microarray analysis of MKN-45 tumor cells to determine the influence of platelets or aspirin.

Results In vivo experiments showed that tumor growth was inhibited by halving the circulating platelet count by using an antiplatelet antibody or peroral daily aspirin. In vitro experiments showed that the proliferation rates of gastric cancer cell lines were increased after coincubation with platelets and that the effect was inhibited by aspirin. Although the expression of interleukin-6, platelet-derived growth factor, transforming growth factor- $\beta$, and prostaglandin E2 did not correlate with tumor growth inhibition by aspirin, seven microRNAs showed altered expression in
\end{abstract}

Electronic supplementary material The online version of this article (doi:10.1007/s10120-015-0556-2) contains supplementary material, which is available to authorized users.

Yukinori Kurokawa

ykurokawa@gesurg.med.osaka-u.ac.jp

1 Department of Gastroenterological Surgery, Osaka University Graduate School of Medicine, 2-2-E2, Yamadaoka, Suita, Osaka 565-0871, Japan cancer cells in response to coincubation with platelets or addition of aspirin. Cells transfected with mir-4670-5p showed a significant increase in proliferation compared to negative control cells.

Conclusions Our study showed that platelets increased the proliferation of gastric cancer cells and that this increase was inhibited by antiplatelet antibody or aspirin. Mir-4670-5p may play an important role in these responses.

Keywords Antiplatelet antibody · Aspirin - Platelet . Mir-4670-5p

\section{Introduction}

Platelets have important functions in hemostasis, immunity, and inflammation, and what is more, they play a key role in cancer growth and metastasis [1]. Since the close relation between platelet count and metastatic activities was first reported in 1968 [2], much experimental evidence has suggested that platelets mediate tumor cell growth, dissemination, and angiogenesis [3]. It is now recognized that tumor cell-induced platelet aggregation (TCIPA) is a crucial mechanism underlying cancer growth and metastasis [4]. Interaction with platelets protects tumor cells from immune elimination and facilitates their survival in blood [5].

Paraneoplastic thrombocytosis is observed in 10-57 \% of patients with cancer, and elevated platelet counts can lead to cancer progression and metastasis [6, 7]. Suppressing platelet activity may improve outcomes in patients with cancer. Several recent studies have investigated treatments with antiplatelet agents, particularly aspirin [3, 8], because aspirin caused a reduction of platelet activities 
[9]. A correlative study of randomized controlled trials evaluating the cardiovascular benefits of aspirin reported that aspirin also reduced the risk of distant metastasis after cancer incidence [8]. This antitumor effect is considered to be driven by the inhibition of cyclooxygenase-2 (COX-2), which is overexpressed in cancer cells $[10,11]$, or by platelet adhesion to tumor cells, the latter of which is essential for preventing immune surveillance by natural killer cells [12]. Stone et al. proposed that the paracrine signal pathway mediates paraneoplastic thrombocytosis in ovarian cancer [7]; interleukin-6 secreted from cancer cells stimulates hepatic thrombopoietin production, which could promote thrombocytosis. However, it is not known whether reducing platelet counts is effective in tumor suppression or which functions of aspirin have antitumor activity in gastric cancer cells. In this study, the possibility of gastric cancer treatment with an antiplatelet agent was examined both in vivo and in vitro.

\section{Materials and methods}

\section{Cell lines and cell culture}

Human gastric cancer cell lines MKN45 (JCRB0254) and NUGC3 (JCRB0822) were obtained from the Japanese Collection of Research Bioresources (Osaka, Japan), and AGS (CRL1739) was obtained from the American Type Culture Collection (Rockville, MD, USA). Cells were cultured in RPMI 1640 (Life Technologies, Carlsbad, CA, USA) medium supplemented with $10 \%$ fetal bovine serum (Sigma-Aldrich, St. Louis, MO, USA) and $1 \%$ penicillin/ streptomycin (Life Technologies) and maintained under an atmosphere of $5 \% \mathrm{CO}_{2}$ at $37{ }^{\circ} \mathrm{C}$.

\section{In vivo experiments}

Male BALB/c nude mice, 4 weeks old, were purchased from CLEA Japan (Tokyo, Japan) and bred in the Animal Laboratory Unit, Osaka University, Japan. A total of $3 \times 10^{6} \mathrm{MKN}-45$ cells in $50 \mu \mathrm{l}$ PBS plus $50 \mu \mathrm{l}$ Matrigel (BD Biosciences, San Jose, CA, USA) were subcutaneously injected into the right flank of each mouse. The mice were randomized into treatment and control groups. For antiplatelet antibody (APA, antimouse glycoprotein $1 b \alpha$, Emfret Analytics, Wurzburg, Germany) experiments, mice were treated by tail vein injection with $100 \mu \mathrm{l}$ APA $(0.5 \mu \mathrm{g} / \mathrm{g}$ of body weight) every 4 days or control $100 \mu \mathrm{l}$ IgG (Emfret Analytics). For aspirin (Sigma-Aldrich) experiments, mice were treated orally with $100 \mu \mathrm{l}$ aspirin (20 mg/kg of body weight) daily or control $100 \mu \mathrm{l}$ drinking water; this aspirin dosage is equivalent to a daily human dosage of about 80-110 mg/day [13]. Aspirin and drinking water were administered using an oral feeding needle. For platelet counts, $30 \mu$ blood was collected from the tail vein prior to cell injection and 2 days after APA injection. Platelet counts were measured using a VetScan HM2 Hematology Analyzer (Abaxis, Union City, CA, USA). Tumor volume was calculated using the equation $V\left(\mathrm{~mm}^{3}\right)=A \times B^{2} / 2$, where $A$ is the largest diameter and $B$ the smallest diameter. All mice were killed on day 22 . The tumors were collected, weighed, and then fixed in formalin and embedded in paraffin. All animal experiments were performed in accordance with the currently prescribed guidelines and using a protocol approved by Osaka University.

\section{Immunohistochemistry}

The expression of $\mathrm{Ki} 67$ and CD31 in the tumors from tumor-bearing mice, fixed as described above, was detected with the DAKO-labeled streptavidin-biotin kit (DAKO, Carpinteria, CA, USA). Slides were boiled in $10 \mathrm{mM}$ citrate buffer ( $\mathrm{pH}$ 6.0) for $20 \mathrm{~min}$ for antigen retrieval and incubated with Ki67 antibody (RM-9106-S, rabbit antihuman monoclonal IgG, diluted 1:200; Thermo Scientific, Waltham, MA, USA) and CD31 antibody (DIA310, rat antimouse monoclonal IgG, diluted 1:20; Dianova, Hamburg, Germany), respectively, followed by the Avidin/Biotin Complex Kit (Vector Laboratories, Burlingame, CA, USA). Tumor tissue was counterstained with hematoxylin after immunostaining. Ki67 staining was recorded as the ratio of positively stained cells to all tumor cells in ten fields from each section at $200 \times$ magnification. Microvessel density (MVD) was the average vessel count in all tumor cells in ten fields from each section at $200 \times$ magnification.

\section{Platelet preparation}

This study was approved by the institutional review board at Osaka University Hospital. For in vitro experiments, platelets were isolated from the venous blood of one healthy volunteer. The platelet preparation method was adapted from Ford et al. [14]. Five vol of OptiPrep ${ }^{\mathrm{TM}}$ was diluted with 22 vol of Solution B [0.85\% (w/v) $\mathrm{NaCl}$, $20 \mathrm{mM}$ Hepes-NaOH, pH 7.4, $1 \mathrm{mM}$ EDTA] to produce a $1.063 \mathrm{~g} / \mathrm{ml}$ density barrier. In a $15-\mathrm{ml}$ centrifuge tube, $5 \mathrm{ml}$ of blood was layered over $5 \mathrm{ml}$ of the $1.063 \mathrm{~g} / \mathrm{ml}$ solution and centrifuged at $350 \mathrm{~g}$ for $15 \mathrm{~min}$ at $20{ }^{\circ} \mathrm{C}$ in a swingingbucket rotor, and the rotor was allowed to decelerate without braking. The platelet-containing band was harvested from the broad turbid band below the interface. Isolated platelets were counted in a Vetscan HM2 Hematology Analyzer (Abaxis) and suspended in RPMI 1640 for concentration adjustment. Cytokines in the culture 
supernatant were measured by enzyme-linked immunosorbent assay (ELISA). Platelet-derived growth factor (R\&D Systems, Minneapolis, MN, USA), transforming growth factor- $\beta$ (R\&D Systems, Minneapolis, MN, USA), and prostaglandin E2 (ENZO Life Science, Famingdale, NY, USA) were measured using an ELISA kit, and interleukin-6 was measured by LSI Medience Corp. (Tokyo, Japan).

\section{CCK-8 assay}

The proliferation rate of human gastric cancer cells was determined by a CCK-8 assay (Dozindo Laboratories, Kumamoto, Japan). The lysed platelets were seeded on porous membranes $(0.4 \mu \mathrm{m}$, PET membrane, Life Technologies) and incubated with gastric cancer cells in a coculture system without direct contact. After 3 days, the membranous insert on which the platelets were seeded was removed, and the CCK-8 solution $(10 \mu \mathrm{l} / 100 \mu \mathrm{l}$ of cell suspension) was added to each well of the plate with the cancer cells. The plate was incubated for $2 \mathrm{~h}$, and the absorbance was then measured at $450 \mathrm{~nm}$ using a microplate reader (Bio-Rad Laboratories, Hercules, CA, USA). Experiments were performed in six wells, and the average was calculated as the value.

\section{MicroRNA microarray}

Total RNA was isolated from cells using TRIzol reagent (Life Technologies) according to the protocol provided by the manufacturer. MicroRNA (miRNA) expression profiling was performed using the 3D-Gene Human miRNA Oligo chip (Toray Industries, Chiba, Japan) with RNA extracted from human MKN-45 gastric cancer cells that were incubated under four different conditions for 3 days: normal conditions, coincubated with platelets only, coincubated with aspirin only, and coincubated with both platelets and aspirin.

\section{MicroRNA transfection}

MKN-45 cells were transfected with pre-miR miRNA precursor molecules at $30 \mathrm{nmol} / \mathrm{l}$ : hsa-miR-485-3p (\#PM10799, Applied Biosystems, Carlsbad, CA, USA), hsa-miR-491-5p (\#PM11479, Applied Biosystems), hsamiR-3200-3p (\#PM17850, Applied Biosystems), hsa-miR4288 (\#PM17022, Applied Biosystems), hsa-miR-4670-5p (\#PM22253, Applied Biosystems), hsa-miR-4725-5p (\#PM22436, Applied Biosystems), and hsa-miR-6887-5p (\#PM27272, Applied Biosystems). Transfection was performed using SiPORT NeoFX (Ambion) in 96-well plates. Pre-miR negative control (\#PM17110, Applied Biosystems) was also used.

\section{Real-time RT-qPCR and TaqMan miRNA assay}

The TaqMan miRNA assay (Applied Biosystems) was used to measure miRNA levels. This assay detects only the mature form of the specific miRNAs. First, $10 \mathrm{ng}$ of RNA was reverse transcribed, and the resulting cDNA was amplified using the following specific TaqMan microRNA assays: hsa-miR-4670-5p, ID 464872; RNU6B, ID 001093. The PCRs were performed with the 7500HT Sequence Detection System (Applied Biosystems). Amplification data were normalized to RNU6B expression. Quantitation of relative expression was conducted using the $2^{-\Delta \Delta \mathrm{Ct}}$ method [15].

\section{Statistical analysis}

In vivo and in vitro analysis, data are expressed as mean \pm standard deviation (SD) ( $n=6$, for each group). Differences between groups were examined for statistical significance by Student's $t$ test. Two-sided $P$ values were calculated, and a value of $P \leq 0.05$ was considered to be statistically significant. All statistical analyses were performed using the SPSS Statistics software program, version 20 (IBM Corp., Armonk, USA).

\section{Results}

\section{In vivo antitumor activity of antiplatelet antibody}

In order to confirm that the platelets were affecting tumor growth in vivo, we used an APA directed against mouse glycoprotein $1 b \alpha$ to reduce platelets in mice bearing gastric cancer tumor MKN-45. Platelet counts were reduced up to $50 \%$ at 2 days after injection with $100 \mu \mathrm{l}$ APA $(0.5 \mu \mathrm{g} / \mathrm{g}$ of body weight) (Fig. S1A), and the reduction was maintained by repeating the injection every 4 days (Fig. S1B). The tumors treated with APA grew more slowly than those treated with control IgG (Fig. 1a). On day 22, tumor weights were lower in tumors treated with APA (Fig. 1b). The proliferation index and MVD in the resected tumors on day 22 were evaluated by staining for Ki67 and CD31 antigens, respectively (Fig. 2a, b). We found that the mice treated with APA had a significantly lower percentage of $\mathrm{Ki} 67$ positivity compared with those treated with control IgG (29.8 vs. $42.7 \%$, respectively; $P<0.05)$. Mice treated with APA demonstrated a significantly greater reduction of MVD, indicative of tumorassociated neovascularization in tumors, than the mice treated with control $\operatorname{IgG}(7.2$ vs. $17.0 \%$, respectively; $P<0.05)$. 

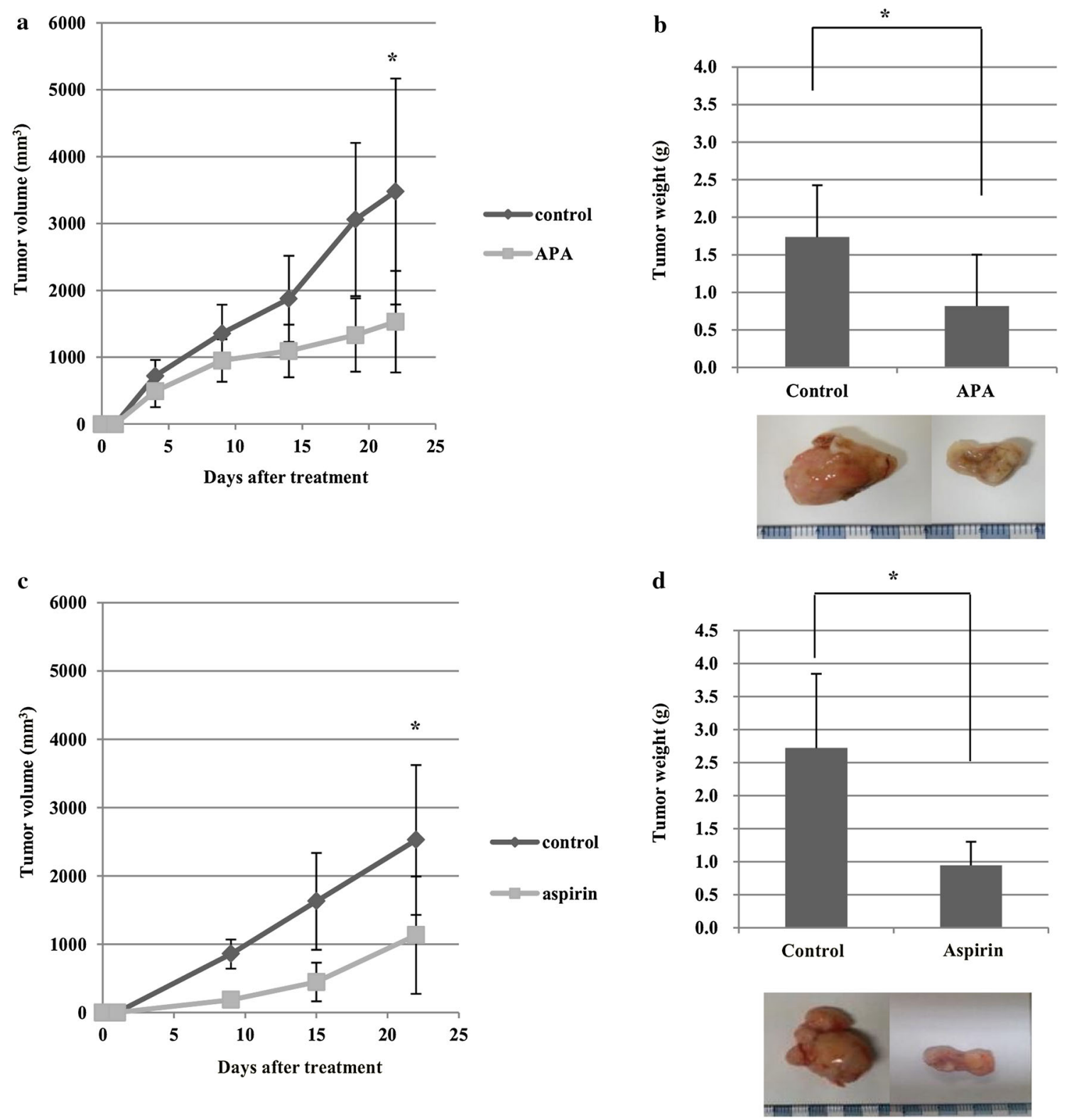

Fig. 1 The antitumor effect of antiplatelet antibody (a, b) and aspirin (c, d) in vivo. Tumor growth, in terms of both size and weight, was significantly inhibited with these agents. ${ }^{*} P<0.05$. The results are mean \pm SD. $n=6$ for each group

\section{In vivo antitumor activity of aspirin}

In order to confirm whether aspirin affects tumor growth in vivo, we used oral aspirin in mice bearing gastric cancer tumor MKN-45. Tumors grew more slowly in mice treated with aspirin than in those treated with water (Fig. 1c). On day 22, tumor weights were lower in mice receiving aspirin (Fig. 1d). The proliferation index and MVD in the resected tumors on day 22 were evaluated by staining for Ki67 and CD31 antigens, respectively. The tumors in mice treated with aspirin showed a lower percentage of Ki67 positivity than those in mice treated with drinking water (33.8 vs. $45.4 \%$, respectively; $P<0.05$ ) (Fig. $2 \mathrm{c}$ ). The aspirin- treated group also showed a greater reduction in MVD (9.5 vs. $14.9 \%$, respectively; $P<0.05$ ) (Fig. 2 d).

\section{In vitro antitumor activity of aspirin}

Human platelets were incubated with MKN-45, NUGC-3, and AGS cells for 3 days. Because platelets and cancer cells had no direct contact due to their separation by the polycarbonate membrane, any platelet effects on cells were due to soluble factors. Platelet concentration $\left(2 \times 10^{7} / \mathrm{ml}\right)$ was according to a previous study [16]; $1 \times 10^{7} / \mathrm{ml}$ of platelets were also used to evaluate the growth effects after halving the concentration. The proliferation rate of all three 


\section{$\mathbf{a}$}
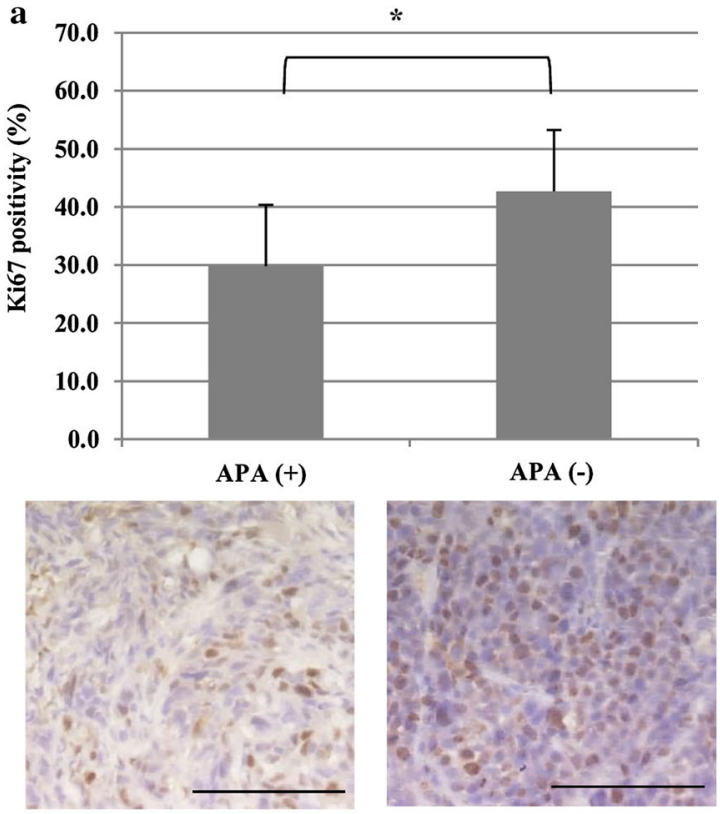

c
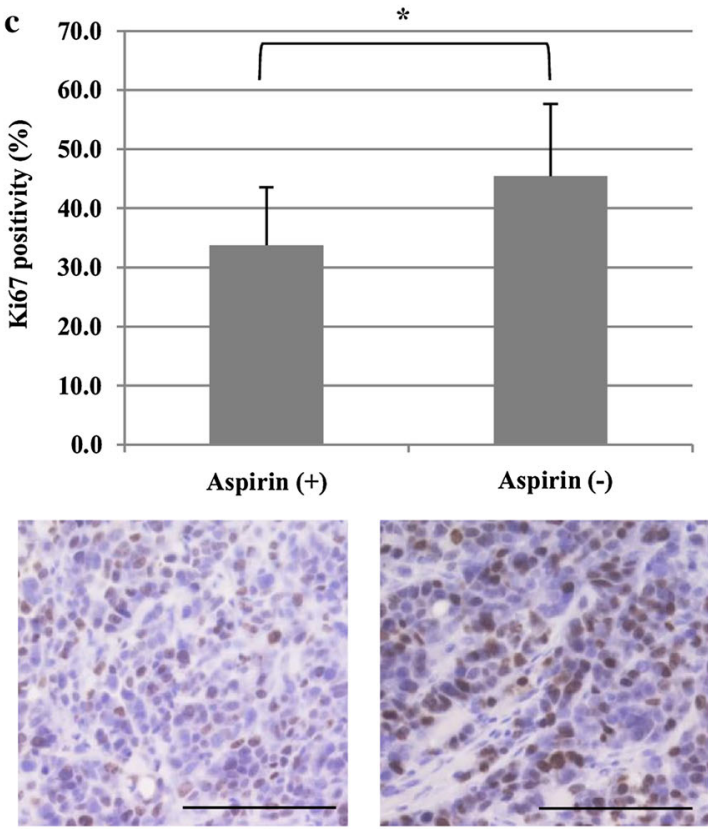

Fig. 2 Immunostaining of tumors resected from mice, using antiplatelet antibody (a, b) or aspirin (c, d). Ki67 positivity (a, c) and MVD (b, d) were lower in mice that received either antiplatelet

types of gastric cancer cells increased after coincubation with platelets (Fig. 3). This effect was more pronounced with higher platelet concentrations.

To confirm that the proliferative effects of platelets were inhibited by aspirin, $1 \mathrm{mM}$ aspirin was added to gastric cancer cells coincubated with $2 \times 10^{7} / \mathrm{ml}$ platelets for three days (Fig. 4), because a previous study showed that $1 \mathrm{mM}$ aspirin caused up to $35 \%$ reduction
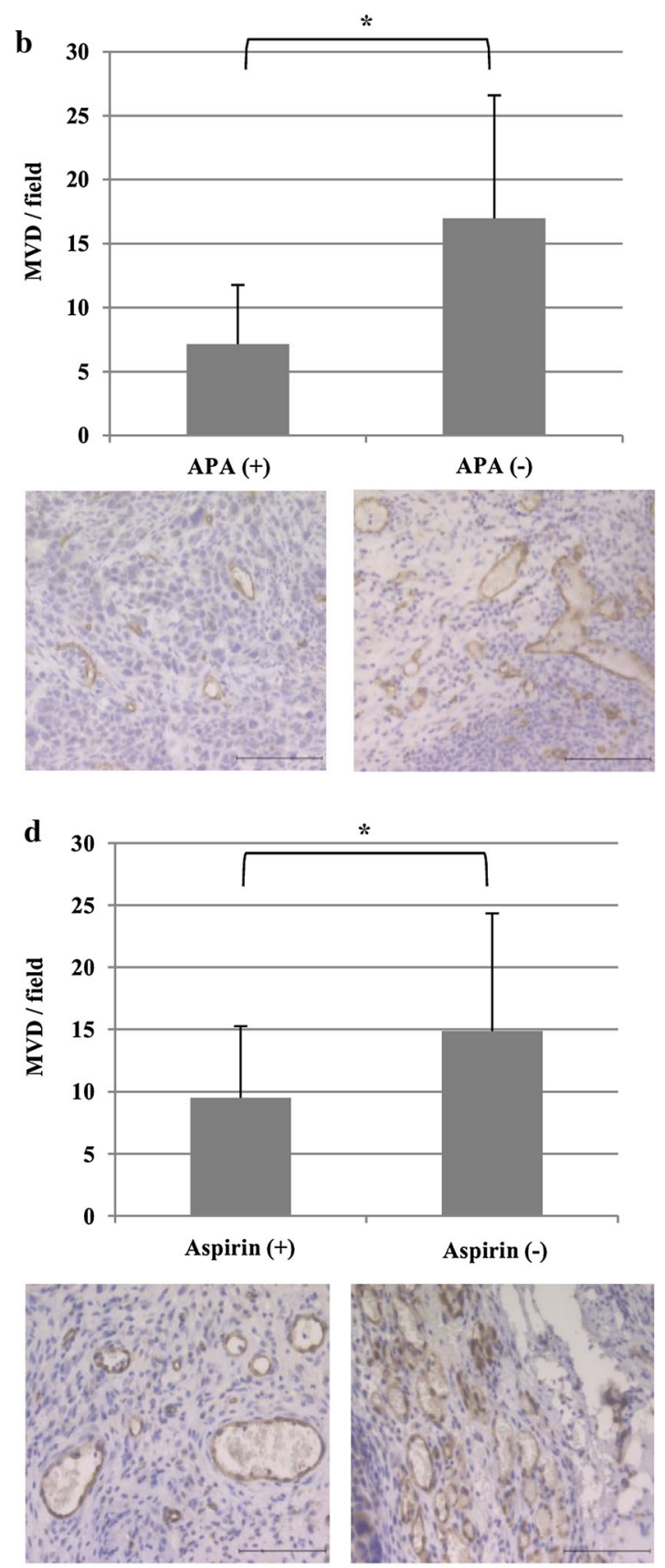

agent. Images were taken at original magnification $\times 100 . * P<0.05$. The results are mean $\pm \mathrm{SD}$. $n=6$ for each group. Scale bars, $100 \mu \mathrm{m}$

of platelet activities [9]. Coincubation with platelets resulted in significant growth of all three types of cancer cells, and this effect was inhibited by aspirin in two of three cell lines. Furthermore, the addition of only aspirin did not alter cell growth in any of the three cell lines.

Because platelets are thought to release numerous factors during activation, and some of those affecting 
MKN-45

$*$

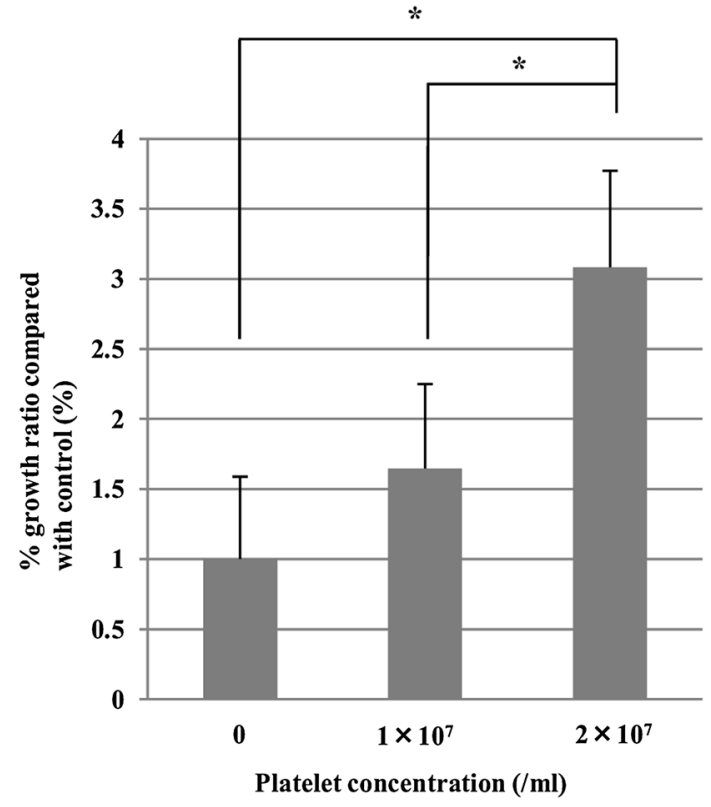

AGS

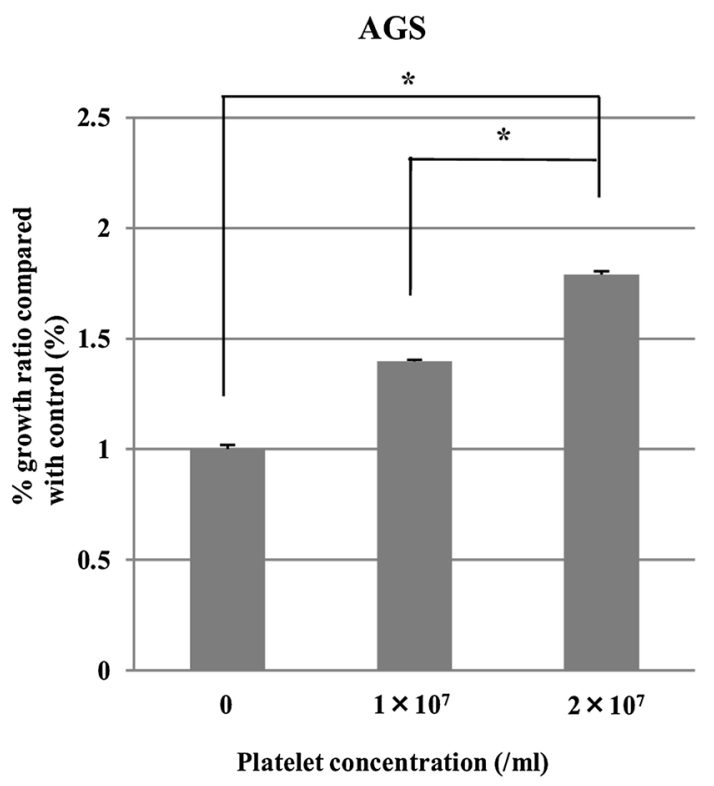

NUGC-3

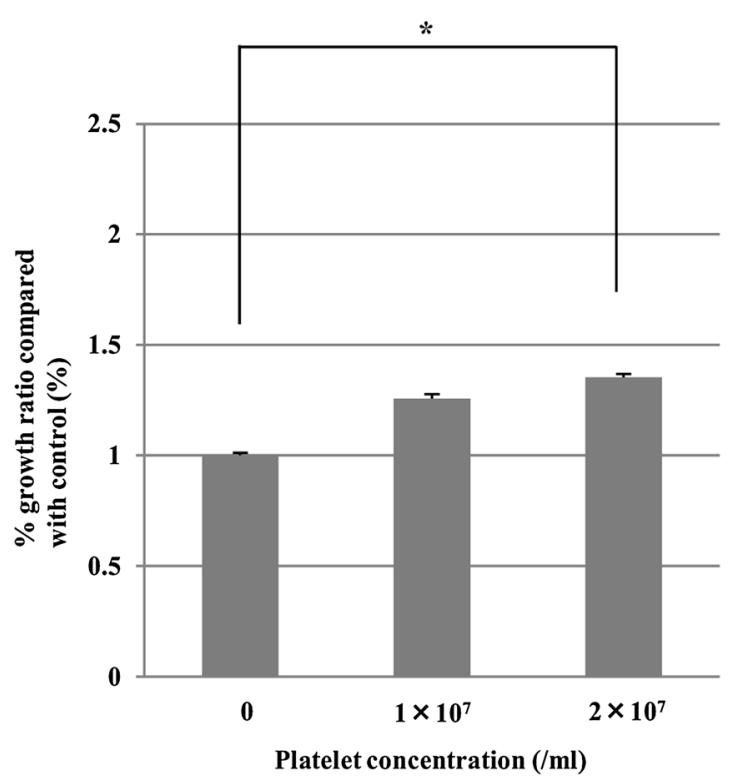

Fig. 3 Proliferative effect of platelets. Results are normalized to cancer cells incubated under normal conditions. Proliferation rates of gastric cancer cells correlated positively with platelet counts. $* P<0.05$. The results are mean \pm SD of six experiments

proliferation can be inhibited by aspirin, we used ELISA assays to examine the effect of four cytokines (interleukin6 , platelet-derived growth factor, transforming growth factor- $\beta$, and prostaglandin E2) on cell proliferation. These cytokines were added to the supernatant in which MKN-45 tumor cells and platelets had been cultured for 3 days either with or without aspirin. None of the cytokines affected aspirin's inhibition of platelet-induced cell growth (Figure S2).

\section{MicroRNA expression analysis}

We examined the miRNA microarray of MKN45 with or without platelets and aspirin to determine the influence of platelets. We examined the following changed miRNAs in cells that had been cultured in four environments: increased more than 1.75 -fold by coincubation with platelets, and the increase was inhibited more than 1.75 -fold by adding aspirin. In total, seven miRNAs (mir-485-3p, mir-491-5p, mir-3200- 

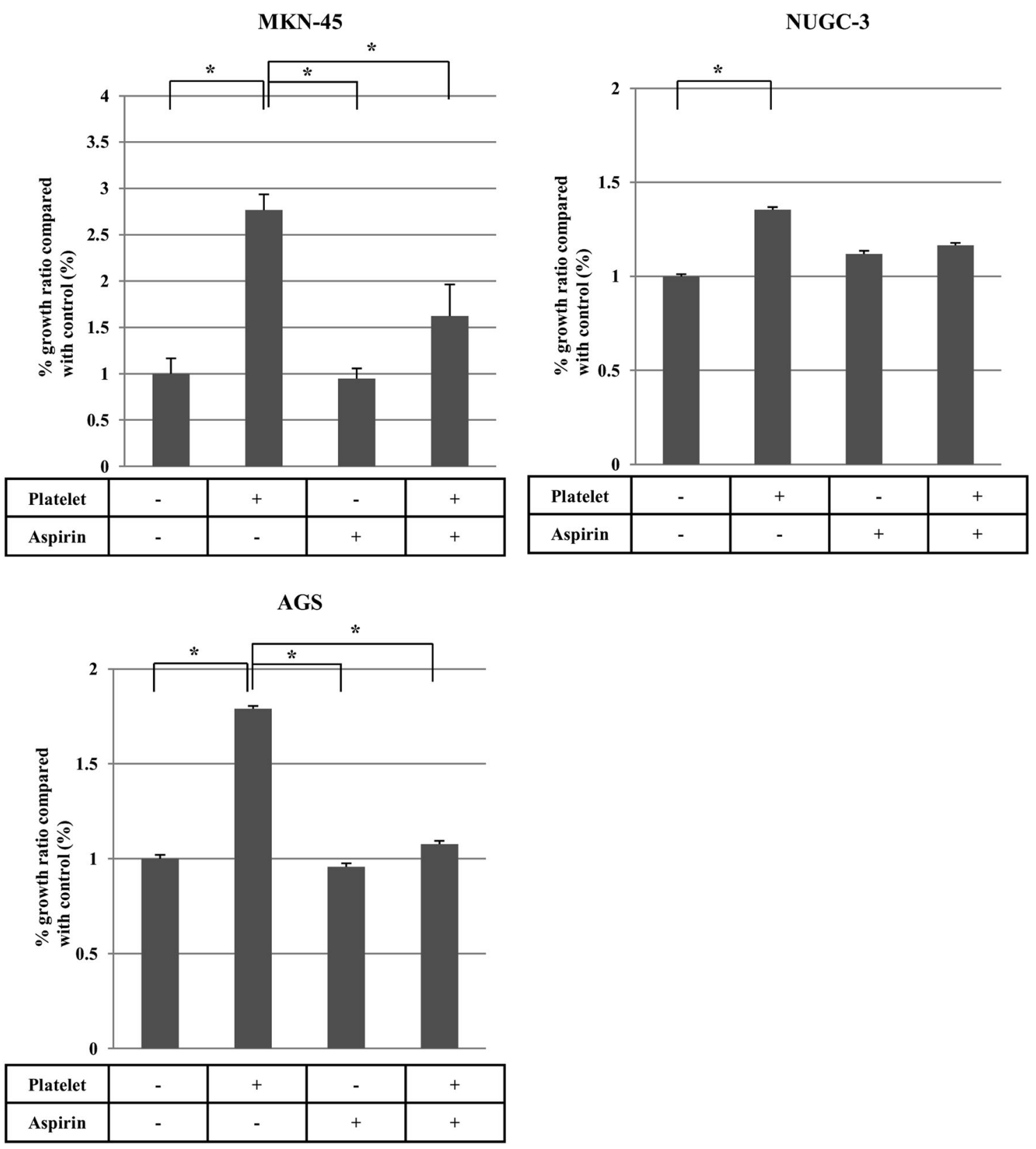

Fig. 4 Proliferative effect of platelets and aspirin in vitro. Results are normalized to cancer cells incubated with RPMI1640 alone. Although the proliferation rates of a MKN-45, b NUGC-3, and c AGS gastric

cancer cells increased after coincubation with platelets, they were inhibited by the addition of $1 \mathrm{mM}$ aspirin. $* P<0.05$. The results are mean $\pm \mathrm{SD}$ of six experiments

3p, mir-4288, mir-4670-5p, mir-4725-5p, mir-6887-5p) were detected with the above-mentioned expression changes (Fig. 5). To confirm which miRNAs were implicated in the response to coincubation with platelets, these miRNAs were transfected into MKN-45 cells. Among them, mir-4670-5ptransfected cells showed a significant increase in the WST assay compared to the negative control cells after day 3 (Figs. 6, S3A, S3B). We confirmed that the expression levels of miR-4670-5p in RT-qPCR were very similar to those in

the microarray (Fig. S4). In vivo experiments showed a clear trend toward suppression of miR-4670-5p expression treated by APA or aspirin (Fig. S5).

\section{Discussion}

In this study, both halving the circulating platelet count and administering daily peroral aspirin inhibited tumor growth in mice, and tumors from mice treated with APA or aspirin 

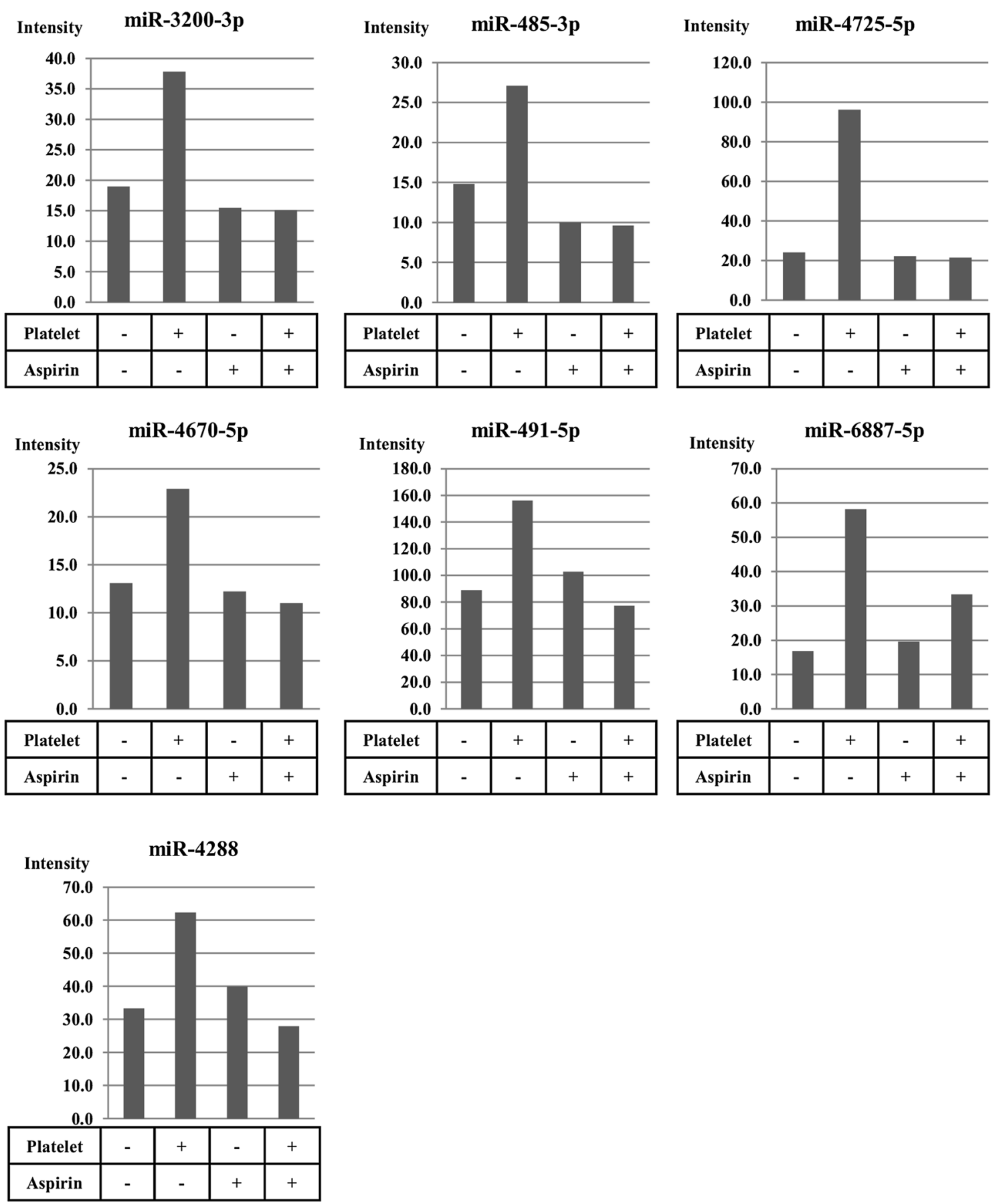

Fig. 5 Fold-changes in MKN-45 gastric cancer cells coincubated with platelets. We examined seven miRNAs whose expression changed in cells cultured under four conditions: with neither platelets

nor aspirin, with platelets only (cell growth increased more than 1.75fold), with aspirin only, and with both platelets and aspirin (cell growth inhibited more than 1.75 -fold)

showed a significantly lower proliferation index (Ki67 positivity) and MVD compared with controls. When tumor cells were coincubated with platelets in vitro in such a way that direct contact was prevented by a polycarbonate membrane, cell growth increased proportionately with platelet count. Although aspirin did not inhibit tumor cell

growth in the absence of platelets, it did inhibit the proliferative effect of platelets.

Thrombocytosis is common in patients with advanced tumors [7, 17], and it is thought that the paraneoplastic increase in platelet count can promote tumor growth. Indeed, among 260 patients with T2-4 gastric cancer who 


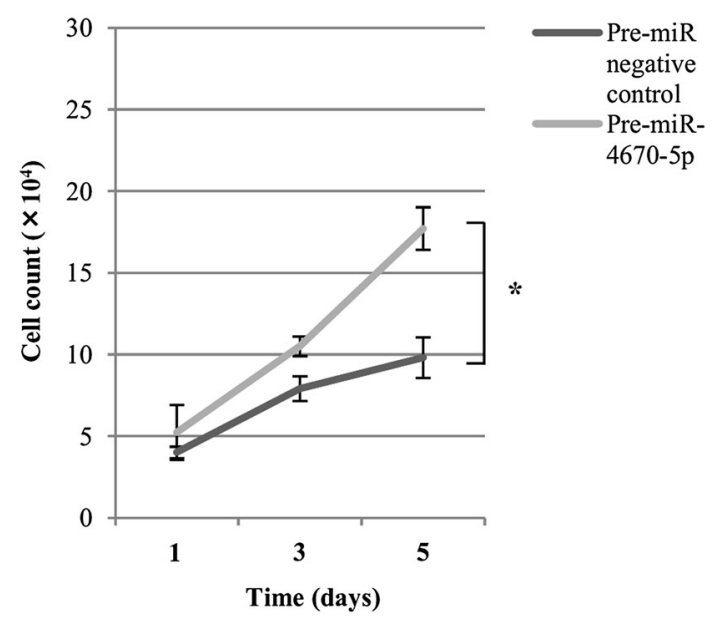

Fig. 6 Overexpression of mir-4670-5p promotes proliferation of gastric cancer cells. Pre-miR-4670-5p treatment led to more growth in MKN-45 cells than in those treated with pre-miR negative control. $* P<0.05$. The results are mean $\pm \mathrm{SD}$ of six experiments

underwent gastrectomy in our hospital, patients with platelet counts over $250,000 / \mu 1$ showed significantly poorer survival compared to those with platelet counts under $250,000 / \mu 1$ (data not shown). While activated platelets release a variety of factors $[5,18]$, it is unknown which are responsible for promoting the proliferation of gastric cancer cells. In our study, microarray analysis showed that seven miRNAs exhibited altered expression in cancer cells in response to coincubation with platelets or the addition of aspirin. In particular, proliferation of mir-4670-5p-transfected cells was significantly higher than that of negative control cells. The change in mir-4670-5p expression in tumor cells was considered a candidate for the antitumor effect through the platelets by aspirin. However, we think several factors such as growth factors or cytokines are involved in tumor growth by the platelet. Since mir-4670$5 p$ might be one of the platelet activities, treatment against not mir-4670-5p but platelets would be more effective.

Several studies have suggested mechanisms underlying the antitumor effects of aspirin, such as prevention of COX-2 overexpression in tumor cells due to inflammation in the absence of platelets or to direct platelet-tumor cell interaction $[10,19]$ or prevention of this interaction by inhibiting cyclooxygenase-1 (COX-1) in platelets [12]. Although COX-1 is expressed constitutively in various tissues, $\mathrm{COX}-2$ is produced by stimulation such as inflammation [20]. COX-2, unlike COX-1, is not produced in the anucleate platelet, whereas cancer cells synthesize COX-2 [10, 21]. Aspirin inhibits both COX-1 and COX-2, but it preferentially inhibits COX-1 [22]. In this study the amount of aspirin we used in mice was equivalent to a low dose in humans [13], which is considered to inhibit tumor growth through platelet inhibition. However, aspirin itself did not demonstrate an anticancer effect and showed a blocking effect at low doses [23]. The model used in the current study suggested that aspirin inhibited tumor growth caused by interaction with platelets.

Several cytokines may be associated with tumor growth induced by platelets and inhibited by aspirin [5]. Although recent studies reported that interleukin-6, platelet-derived growth factor, transforming growth factor- $\beta$, and prostaglandin E2 might affect tumor growth [7, 24, 25], those we examined did not exhibit such activity. Platelets are anucleate but do have messenger RNA, whose translation may be regulated by miRNAs [26]. Although there are no reports of miRNA associated with tumor growth induced by platelets, our findings showed that mir-4670-5p may play such a role. One of the transcripts of mir-4670-5p is calmodulin-binding transcription activator 1 (CAMTA1). Because the previous studies showed that CAMTA1 inhibits tumor progression in some cancers [21, 27], the excessive mir-4670-5p in tumor cells due to the interaction with platelets may inhibit the expression of CAMTA1 and lead to the progression of tumor cells. However, to our knowledge, there are no reports demonstrating the significance of mir-4670-5p in cancer cells, and thus further investigation is needed to elucidate its function.

In conclusion, our in vivo and in vitro experiments showed that platelets increased the proliferation of gastric cancer cells and that this increase was inhibited by aspirin. In addition, mir-4670-5p may play an important role in these processes.

\section{Compliance with ethical standards}

Conflict of interest The authors declare that they have no competing interests. All institutional and national guidelines for the care and use of laboratory animals were followed.

\section{References}

1. Sharma D, Brummel-Ziedins KE, Bouchard BA, Holmes CE. Platelets in tumor progression: a host factor that offers multiple potential targets in the treatment of cancer. J Cell Physiol. 2014;229:1005-15.

2. Gasic GJ, Gasic TB, Stewart CC. Antimetastatic effects associated with platelet reduction. Proc Natl Acad Sci U S A. 1968;61:46-52.

3. Bambace NM, Holmes CE. The platelet contribution to cancer progression. J Thromb Haemost. 2011;9:237-49.

4. Goubran HA, Burnouf T, Radosevic M, El-Ekiaby M. The platelet-cancer loop. Eur J Intern Med. 2013;24:393-400.

5. Gay LJ, Felding-Habermann B. Contribution of platelets to tumour metastasis. Nat Rev Cancer. 2011;11:123-34.

6. Sierko E, Wojtukiewicz MZ. Platelets and angiogenesis in malignancy. Semin Thromb Hemost. 2004;30:95-108.

7. Stone RL, Nick AM, McNeish IA, Balkwill F, Han HD, Bottsford-Miller J, et al. Paraneoplastic thrombocytosis in ovarian cancer. N Engl J Med. 2012;366:610-8. 
8. Rothwell PM, Wilson M, Price JF, Belch JF, Meade TW, Mehta Z. Effect of daily aspirin on risk of cancer metastasis: a study of incident cancers during randomised controlled trials. Lancet. 2012;379:1591-601.

9. Begent NA, Born GV. Comparison of the effects of drugs on the aggregation of hamster platelets in vivo and in vitro. Br J Pharmacol. 1971;43:580-92.

10. Cathomas G. PIK3CA in Colorectal Cancer. Front Oncol. 2014;4:35.

11. Okumura H, Uchikado Y, Setoyama T, Matsumoto M, Owaki T, Ishigami $\mathrm{S}$, et al. Biomarkers for predicting the response of esophageal squamous cell carcinoma to neoadjuvant chemoradiation therapy. Surg Today. 2014;44:421-8.

12. Reimers MS, Bastiaannet E, Langley RE, van Eijk R, van Vlierberghe RL, Lemmens VE, et al. Expression of HLA class I antigen, aspirin use, and survival after a diagnosis of colon cancer. JAMA Intern Med. 2014;174:732-9.

13. Mahmoud NN, Dannenberg AJ, Mestre J, Bilinski RT, Churchill MR, Martucci C, et al. Aspirin prevents tumors in a murine model of familial adenomatous polyposis. Surgery. 1998;124:225-31.

14. Ford TC, Graham J, Rickwood D. A new, rapid, one-step method for the isolation of platelets from human blood. Clin Chim Acta. 1990;192:115-9.

15. Livak KJ, Schmittgen TD. Analysis of relative gene expression data using real-time quantitative PCR and the 2[-Delta Delta $\mathrm{C}(\mathrm{T})$ ] method. Methods. 2001;25:402-8.

16. Cho MS, Bottsford-Miller J, Vasquez HG, Stone R, Zand B, Kroll $\mathrm{MH}$, et al. Platelets increase the proliferation of ovarian cancer cells. Blood. 2012;120:4869-72.

17. Ikeda M, Furukawa H, Imamura H, Shimizu J, Ishida H, Masutani $\mathrm{S}$, et al. Poor prognosis associated with thrombocytosis in patients with gastric cancer. Ann Surg Oncol. 2002;9:287-91.
18. Takahashi K, Murata S, Ohkohchi N. Novel therapy for liver regeneration by increasing the number of platelets. Surg Today. 2013;43:1081-7.

19. Dovizio M, Maier TJ, Alberti S, Di Francesco L, Marcantoni E, Munch G, et al. Pharmacological inhibition of platelet-tumor cell cross-talk prevents platelet-induced overexpression of cyclooxygenase-2 in HT29 human colon carcinoma cells. Mol Pharmacol. 2013;84:25-40.

20. Uddin S, Ahmed M, Hussain A, Assad L, Al-Dayel F, Bavi P, et al. Cyclooxygenase-2 inhibition inhibits PI3 K/AKT kinase activity in epithelial ovarian cancer. Int J Cancer. 2010;126:382-94.

21. Henrich KO, Bauer T, Schulte J, Ehemann V, Deubzer H, Gogolin S, et al. CAMTA1, a 1p36 tumor suppressor candidate, inhibits growth and activates differentiation programs in neuroblastoma cells. Cancer Res. 2011;71:3142-51.

22. Langley RE, Burdett S, Tierney JF, Cafferty F, Parmar MK, Venning G. Aspirin and cancer: has aspirin been overlooked as an adjuvant therapy? Br J Cancer. 2011;105:1107-13.

23. Goel A, Chang DK, Ricciardiello L, Gasche C, Boland CR. A novel mechanism for aspirin-mediated growth inhibition of human colon cancer cells. Clin Cancer Res. 2003;9:383-90.

24. Heldin $\mathrm{CH}$. Targeting the PDGF signaling pathway in tumor treatment. Cell Commun Signal. 2013;11:97.

25. Labelle M, Begum S, Hynes RO. Direct signaling between platelets and cancer cells induces an epithelial-mesenchymal-like transition and promotes metastasis. Cancer Cell. 2011;20:576-90.

26. Landry P, Plante I, Ouellet DL, Perron MP, Rousseau G, Provost P. Existence of a microRNA pathway in anucleate platelets. Nat Struct Mol Biol. 2009;16:961-6.

27. Schraivogel D, Weinmann L, Beier D, Tabatabai G, Eichner A, Zhu JY, et al. CAMTA1 is a novel tumour suppressor regulated by miR-9/9* in glioblastoma stem cells. EMBO J. 2011;30:4309-22. 